\title{
CONTROLLED HOMOTOPY EQUIVALENCES AND STRUCTURE SETS OF MANIFOLDS
}

\author{
FRIEDRICH HEGENBARTH AND DUŠAN REPOVS̆
}

(Communicated by Alexander N. Dranishnikov)

\begin{abstract}
For a closed topological $n$-manifold $K$ and a map $p: K \rightarrow B$ inducing an isomorphism $\pi_{1}(K) \rightarrow \pi_{1}(B)$, there is a canonically defined morphism $b: H_{n+1}(B, K, \mathbb{L}) \rightarrow \mathcal{S}(K)$, where $\mathbb{L}$ is the periodic simply connected surgery spectrum and $\mathcal{S}(K)$ is the topological structure set. We construct a refinement $a: H_{n+1}^{+}(B, K, \mathbb{L}) \rightarrow \mathcal{S}_{\varepsilon, \delta}(K)$ in the case when $p$ is $U V^{1}$, and we show that $a$ is bijective if $B$ is a finite-dimensional compact metric ANR. Here, $H_{n+1}^{+}(B, K, \mathbb{L}) \subset H_{n+1}(B, K, \mathbb{L})$, and $\mathcal{S}_{\varepsilon, \delta}(K)$ is the controlled structure set. We show that the Pedersen-Quinn-Ranicki controlled surgery sequence is equivalent to the exact $\mathbb{L}$-homology sequence of the map $p: K \rightarrow B$, i.e. that

$$
H_{n+1}(B, \mathbb{L}) \rightarrow H_{n+1}^{+}(B, K, \mathbb{L}) \rightarrow H_{n}\left(K, \mathbb{L}^{+}\right) \rightarrow H_{n}(B, \mathbb{L}), \mathbb{L}^{+} \rightarrow \mathbb{L},
$$

is the connected covering spectrum of $\mathbb{L}$. By taking for $B$ various stages of the Postnikov tower of $K$, one obtains an interesting filtration of the controlled structure set.
\end{abstract}

\section{INTRODUCTION}

Let $K$ denote a closed topological manifold of dimension $n$. As usual, $\mathcal{S}(K)$ denotes the topological structure set of $K$. Elements of $\mathcal{S}(K)$ are equivalence classes of pairs $(M, h)$, where $M$ is a closed topological $n$-manifold and $h: M \rightarrow K$ is a simple homotopy equivalence. The pairs $(M, h),\left(M_{1}, h_{1}\right)$ are equivalent if there is a homeomorphism $\varphi: M_{1} \rightarrow M$ such that $h \circ \varphi$ is homotopic to $h_{1} . \mathcal{S}(K)$ has a group structure. If $B$ is a finite-dimensional compact metric ANR and $p: K \rightarrow B$ a continuous $U V^{1}$ map, the controlled structure set $\mathcal{S}_{\varepsilon, \delta}(K \rightarrow B)$ (cf. $\mathrm{PQR}$ ) of $\delta$-homotopy equivalences can be defined (see precise definitions below). There is an obvious forgetful map $\mathcal{S}_{\varepsilon, \delta}(K \rightarrow B) \rightarrow \mathcal{S}(K)$. An element of the image will be called a controlled homotopy equivalence $h: M \rightarrow K$ with respect to $p$. Varying $p: K \rightarrow B$, we get the subset of controlled homotopy equivalences in $\mathcal{S}(K)$.

Dranishnikov and Ferry studied the subset (indeed a subgroup) $\mathcal{S}^{C E}(K) \subset \mathcal{S}(K)$ of elements realized by cell-like maps (cf. [DrFe]). They observed that these elements belong to the image of a natural map

$$
b: H_{n+1}\left(E_{2}(K), K ; \mathbb{L}\right) \rightarrow \mathcal{S}(K)
$$

localized away from 2 .

Received by the editors October 28, 2010 and, in revised form, December 32012.

2010 Mathematics Subject Classification. Primary 57R67, 57P10, 57R65; Secondary 55N20, $55 \mathrm{M} 05$.

Key words and phrases. Controlled surgery, $U V^{1}$-property, $\mathbb{L}$-homotopy, $\mathbb{L}$-homology, controlled structure set, Wall obstruction. 
Here $\mathbb{L}$ is the simply connected 4-periodic surgery spectrum, and $E_{2}(K)$ is the second stage of the Postnikov tower of $K$. The map $b$ can be defined using the diagram on p. 207 of $\mathrm{Ran}$. The main ingredient is the $\pi-\pi$-theorem. The morphism $b$ can be defined for any map $p: K \rightarrow B$ satisfying $p_{*}: \pi_{1}(K) \cong \pi_{1}(B)$. We shall generically call it $b$.

One may ask of what type are homotopy equivalences of images of other Postnikov stages. In this paper we shall give an answer in the case of the first stage, i.e. $B \pi$, where $\pi=\pi_{1}(K)$. As a consequence of the main theorem we shall get the following result:

Theorem 1. Let $n \geq 5$. Then the image of the map

$$
b: H_{n+1}(B \pi, K, \mathbb{L}) \rightarrow \mathcal{S}(K)
$$

contains the controlled homotopy equivalences.

In order to be able to state the main result we shall need some more notations. Recall that $\mathbb{L}_{0}=\mathbb{Z} \times G / T O P$. Let $\mathbb{L}^{+} \rightarrow \mathbb{L}$ be the simply connected spectrum covering $\mathbb{L}$. In particular, $\mathbb{L}_{0}^{+}=G / T O P$. For any pair $(Z, Y)$ there is the following exact sequence (cf. [Ran, p. 153]):

$(*) \quad \cdots \rightarrow H_{m+2}(Z, Y, \mathbb{Z}) \rightarrow H_{m+1}\left(Z, Y, \mathbb{L}^{+}\right) \rightarrow H_{m+1}(Z, Y, \mathbb{L}) \rightarrow H_{m+1}(Z, Y, \mathbb{Z}) \rightarrow \cdots$.

In particular, for $(K, \emptyset)$, where $K$ is an oriented closed topological $n$-manifold, we have an injection

$$
H_{n}\left(K, \mathbb{L}^{+}\right) \rightarrow H_{n}(K, \mathbb{L}) .
$$

Let us denote the inverse image of $H_{n}\left(K, \mathbb{L}^{+}\right)$under $\partial_{*}: H_{n+1}(B, K, \mathbb{L}) \rightarrow$ $H_{n}(K, \mathbb{L})$ by $H_{n+1}^{+}(B, K, \mathbb{L}) \subset H_{n+1}(B, K, \mathbb{L})$. Here $B$ is a space containing $K$, or more generally, a map $K \rightarrow B$.

Theorem 2. Suppose that $p: K \rightarrow B$ is a $U V^{1}$-map into a compact metric ANRspace, with $K$ as above and $n \geq 5$. Then there is a map

$$
a: H_{n+1}^{+}(B, K, \mathbb{L}) \rightarrow \mathcal{S}_{\varepsilon, \delta}(K \rightarrow B),
$$

where $\varepsilon, \delta$ are appropriately choosen (see below). The map a fits into the following diagram:

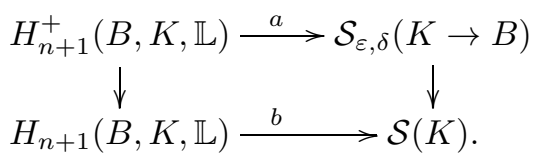

The natural map $H_{n+1}(B, \mathbb{L}) \rightarrow H_{n+1}(B, K, \mathbb{L})$ factors over $H_{n+1}^{+}(B, K, \mathbb{L})$, and one can easily see that the sequence

$$
\cdots \rightarrow H_{n+1}(B, \mathbb{L}) \rightarrow H_{n+1}^{+}(B, K, \mathbb{L}) \rightarrow H_{n}\left(K, \mathbb{L}^{+}\right) \rightarrow H_{n}(B, \mathbb{L}) \rightarrow \cdots
$$

is exact.

Recall that by the Poincaré duality $H_{n}\left(K, \mathbb{L}^{+}\right) \cong[K, G / T O P]$.

Theorem 3. Let $K$ be a closed oriented topological $n$-manifold, $n \geq 5$, and let $p: K \rightarrow B$ be a $U V^{1}$-map into a finite-dimensional compact metric ANR. Then 
there exists a commutative diagram of exact sequences

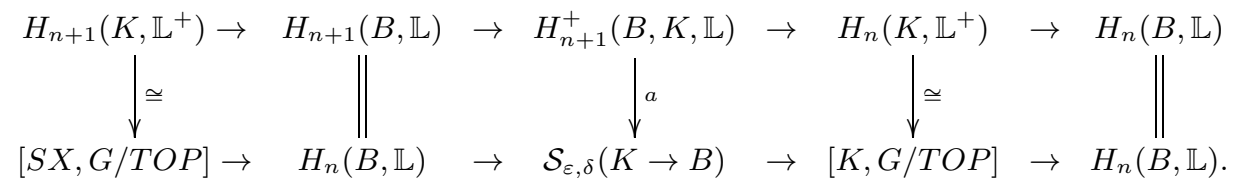

In particular, a is bijective.

Here the lower exact sequence is the controlled surgery sequence from $[\mathrm{PQR}$ and $\varepsilon$ and $\delta$ are to be appropriately chosen: There is an $\varepsilon_{0}>0$, depending on $B$ and $n$, such that for any $0<\varepsilon<\varepsilon_{0}$ there is $\delta>0$ such that the lower sequence is exact.

Theorem 2 and Theorem 3 will be proved in $\S 5$ and $\S 6$, respectively. Theorem 1 follows from Theorem 2, since any $U V^{1}$-map $K \rightarrow B$ composed with the canonical map $B \rightarrow B \pi$ determines a map $(B, K) \rightarrow(B \pi, K)$. Since $K$ is a manifold of dimension $\geq 5$ we can assume that $K \rightarrow B \pi$ is $U V^{1}$ (cf. BFMW]). Hence we get an induced morphism

$$
H_{n+1}(B, K, \mathbb{L}) \rightarrow H_{n+1}(B \pi, K, \mathbb{L}) .
$$

On the other hand, any $[y] \in H_{n+1}(B \pi, K, \mathbb{L})$ has a compact support $(B, K) \subset$ $(B \pi, K)$ (cf. $[\mathrm{Ran}, \S 12])$, and we may assume $K \rightarrow B$ is $U V^{1}$.

To define the map $a$ we recall the geometric $\mathbb{L}$-spectrum, and represent elements $[x] \in H_{n}(K, \mathbb{L})$ and $[y] \in H_{n+1}(B, K, \mathbb{L})$ in terms of normal degree 1 maps. We shall follow $\mathrm{Qu1}$, Nic and $\mathrm{Ran}$. One reason to recall these is to stabilize the notations used in the proofs. Another reason is that Ran used the algebraic $\mathbb{L}$-spectrum defined by quadratic Poincaré chain complexes which led to representing $[x]$ by a normal degree 1 map between closed manifolds (cf. Corollary 18.6(I) in [Ran]). The gluing construction using the geometric $\mathbb{L}$-spectrum gives surgery problems with homotopy equivalences on boundaries. Explicit examples have recently been given (cf. [Ham, Example 5.4]).

\section{The PERIODIC SIMPLY CONNECTED L-SPECTRUM}

We briefly recall the semi-simplicial surgery spectrum introduced in Qu1 (cf. also [Nic]), denoted by $\mathbb{L}=\left\{\mathbb{L}_{g} \mid g \in \mathbb{Z}\right\}$. It is an $\Omega$-spectrum, and each $\mathbb{L}_{g}$ satisfies the Kan condition. Its homotopy groups are the Wall groups of the trivial fundamental group, more precisely, $\pi_{j}\left(\mathbb{L}_{g}\right)=L_{j+g}(\{1\})$, and $\mathbb{L}_{0}=\mathbb{Z} \times G / T O P$.

Let $\mathbb{L}_{g}(j)$ denote the $j$-simplices of $\mathbb{L}_{g}$. An element $x$ in $\mathbb{L}_{g}(j)$ is a degree 1 normal map of $(j+3)$-ads, which we shall shortly denote by

$$
x=\{(f, b): M \rightarrow X\},
$$

where $\left(M, \partial_{0} M, \ldots, \partial_{j} M, \partial_{j+1} M\right)\left(\operatorname{resp} .\left(X, \partial_{0} X, \ldots, \partial_{j} X, \partial_{j+1} X\right)\right)$ are the $(j+3)$ ads $M$ (resp. $X)$. It is required that $\left.f\right|_{\partial_{j+1} M}$ is a (simple) homotopy equivalence. $M$ and $X$ are topological manifolds of dimension $j+g$ with boundaries $\partial X=\cup_{i=0}^{j+1} \partial_{i} X$ and $\partial M=\cup_{i=0}^{j+1} \partial_{i} M$. To $x$ belongs a reference map from $X$ to the $(j+3)$-ad $\Delta^{j}$, i.e. a map $\left(X, \partial_{0} X, \ldots, \partial_{j} X, \partial_{j+1} X\right) \rightarrow\left(\Delta^{j}, \partial_{0} \Delta^{j}, \ldots, \partial_{j} \Delta^{j}, \Delta^{j}\right)$. We emphasize that the condition that $\left.f\right|_{\partial_{j+1} M}: \partial_{j+1} M \rightarrow \partial_{j+1} X$ is a homotopy equivalence is necessary to indentify the homotopy groups of $\mathbb{L}$ with the Wall groups.

The Cartesian product with $\mathbb{C} P^{2}$ defines a semi-simplicial map $\mathbb{L}_{g} \rightarrow \mathbb{L}_{g+4}$ which is a homotopy equivalence, called the periodicity map. 


\section{L-HOMOLOGY CLASSES}

We shall follow $\underline{\operatorname{Ran}}, \S 12]$, to describe elements $[x] \in H_{n}(K, \mathbb{L})$, where $K$ is a finite ordered simplicial complex. The relative case will be given in $\S 4$.

The class $[x]$ is represented by a "cycle" $x$, which roughly is a semi-simplicial map from $K$ to $\mathbb{L}_{g}$ for some $g$. More precisely, one embeds $K$ into $\partial \Delta^{m+1}, \Delta^{m+1}$ the standard $(m+1)$-simplex with vertices denoted $\{0,1, \ldots, m+1\}$. The embedding is order-preserving and one may assume $m+1=$ number of vertices of $K$. For any $\sigma \in \partial \Delta^{m+1}$, let $D\left(\sigma, \partial \Delta^{m+1}\right)$ be the dual cell, and let $\Sigma^{m}$ be the dual cell-complex decomposition of $\partial \Delta^{m+1}$. Note that $D\left(\sigma, \partial \Delta^{m+1}\right)$ is an $(m-|\sigma|)$-ball with boundaries $\partial_{i} D\left(\sigma, \partial \Delta^{m+1}\right)=D\left(\delta_{i} \sigma, \partial \Delta^{m+1}\right)$, where $\sigma=\left\{j_{0}, \ldots, j_{k}\right\} \subset\{0, \ldots, m+1\}$, and $\delta_{i} \sigma=\sigma \cup\left\{\lambda_{i}\right\}$ with $\{0, \ldots, m+1\} \backslash\left\{j_{0}, \ldots, j_{h}\right\}=\left\{\lambda_{0}, \ldots, \lambda_{m-k}\right\}$ (written in that order $)$. Hence $\left\{D\left(\sigma, \partial \Delta^{m+1}\right), \partial_{0} D\left(\sigma, \partial \Delta^{m+1}\right), \ldots, \partial_{m-|\sigma|} D\left(\sigma, \partial \Delta^{m+1}\right)\right\}$ is an $(m-|\sigma|+2)$-ball-ad.

Define $\bar{K}=\left\{D\left(\sigma, \partial \Delta^{m+1}\right) \mid \sigma \in \partial \Delta^{m+1} \backslash K\right\}$ and call it the supplement of $K$. The cycle $x$ can be considered as a semi-simplicial map

$$
x:\left(\Sigma^{m}, \bar{K}\right) \rightarrow\left(\mathbb{L}_{n-m}, \emptyset\right) ;
$$

i.e. $x\left(D\left(\sigma, \partial \Delta^{m+1}\right)\right) \in \mathbb{L}_{n-m}(m-|\sigma|)$ is a surgery problem of $(m-|\sigma|+3)$-ads denoted simply

$$
x=\left\{x_{\sigma}=\left(f_{\sigma}, b_{\sigma}\right): M_{\sigma} \rightarrow X_{\sigma} \mid \sigma \in \partial \Delta^{m+1}\right\}
$$

between manifold $(m-|\sigma|+3)$-ads of dimension $n-|\sigma|$ together with a reference map of $(n-|\sigma|+3)$-ads

$$
\begin{gathered}
\left(X_{\sigma}, \partial_{0} X_{\sigma}, \ldots, \partial_{m-|\sigma|} X_{\sigma}, \partial_{m-|\sigma|+1} X_{\sigma}\right) \\
\rightarrow\left(D\left(\sigma, \partial \Delta^{m+1}\right), \partial_{0} D\left(\sigma, \partial \Delta^{m+1}\right), \ldots, \partial_{m-|\sigma|} D\left(\sigma, \partial \Delta^{m+1}\right), D\left(\sigma, \partial \Delta^{m+1}\right)\right) .
\end{gathered}
$$

Moreover, $f_{\sigma}$ restricted to $\partial_{m-|\sigma|+1} M_{\sigma} \rightarrow \partial_{m-|\sigma|+1} X_{\sigma}$ is a homotopy equivalence. Note that $X_{\sigma} \neq \emptyset \Rightarrow \sigma \in K$.

If $x^{\prime}=\left\{x_{\sigma}^{\prime} \mid \sigma \in \partial \Delta^{m+1}\right\}$ is a cycle defining the same class as $x$, there is a semi-simplicial map

$$
y=\Delta^{1} \times\left(\Sigma^{m}, \bar{K}\right) \rightarrow\left(\mathbb{L}_{n-m}, \emptyset\right)
$$

such that $y\left(\partial_{0} \Delta^{1} \times \sigma\right)=x(\sigma), y\left(\partial_{1} \Delta^{1} \times \sigma\right)=x^{\prime}(\sigma)$.

Next we assemble the pieces $\left\{X_{\sigma}\right\}$, resp. $\left\{M_{\sigma}\right\}$; i.e. we build colimits. For this we use results from LaMc which are elegant reformulation of BRS.

Let $A_{S T O P}$ be the graded category with objects $n$-dimensional oriented topological manifolds with boundaries. The morphisms are orientation preserving inclusions (if dimension preserving) or inclusions with the image in boundaries (if dimension increasing). We refer to Example 3.5 from [LaMc].

Given the cycle $x$ as above we obtain two functors $\left(\Sigma^{m}, \bar{K}\right) \rightarrow\left(A_{S T O P}, \emptyset\right)$ of degree $m-n$, namely we associate to $x(\sigma)$ either the target or the domain: i.e.

$$
D\left(\sigma, \partial \Delta^{m+1}\right) \rightarrow X_{\sigma} \text { and } D\left(\sigma, \partial \Delta^{m+1}\right) \rightarrow M_{\sigma} .
$$

Let $X$, resp. $M$, denote its colimits, and denote by $(f, b): M \rightarrow X$ the resulting map. By Proposition 6.6 of [ $\mathrm{LaMc}, M$ and $X$ are $n$-manifolds with boundaries $\partial M$, $\partial X$, respectively. In fact, $\partial M$ and $\partial X$ are the colimits of the restricted functors to the boundary components $\partial_{m-|\sigma|+1} M_{\sigma}$, resp. $\partial_{m-|\sigma|+1} X_{\sigma}$.

There are no more boundary components. To see this we may consider a (simplicial) neighborhood $N(K)$ of $K \subset \partial \Delta^{m+1}$. Then $X_{\sigma}=\emptyset$ for all $\sigma \notin K$; in particular $X_{\sigma}=\emptyset$ if $\sigma \in \partial N(K)$. 
Together with the colimit of the reference maps one has obtained a normal degree 1 map $(f, b): M \rightarrow X$ with a reference map $\varphi: X \rightarrow N(K) \rightarrow K$, where $N(K) \rightarrow K$ is a retraction.

Remarks.

(I) The restriction of $f$ to $\partial M$ is a homotopy equivalence. Indeed, it is a $\delta$-homotopy equivalence. This can be seen by applying the gluing construction (cf. e.g. [Hat, Ch. 4.G]) inductively to the family $\left\{f_{\sigma} \mid: \partial_{m-|\sigma|+1} M_{\sigma} \rightarrow\right.$ $\left.\partial_{m-|\sigma|+1} X_{\sigma} \mid \sigma \in K\right\}$. Here $\delta$ can be chosen as small as necessary by taking a sufficiently fine subdivision.

(II) A careful construction shows that $\varphi$ is transverse to all $D(\sigma, K)$, the duals of $\sigma$ in $K$, and that

$$
\varphi^{-1}(D(\sigma, K))=X_{\sigma} .
$$

Conversely, any simplicial map from a manifold $\varphi: X \rightarrow K$ is transversal to dual cells $(\underline{\mathrm{Coh}})$. Hence any normal degree 1 map $M \rightarrow X$ defines a class $[x] \in H_{n}(K, \mathbb{L})$.

(III) If $K$ is not a finite complex, then one can choose for any $[x] \in H_{n}(K, \mathbb{L})$ a finite complex $J \subset K$, so that $[x]$ belongs to the image of $H_{n}(J, \mathbb{L}) \rightarrow$ $H_{n}(K, \mathbb{L})$.

(IV) Instead of Proposition 6.6 from [LaMc], one can invoke the gluing procedure applied in [Nic, Ch. 3]. However, the notion of degree decreasing functors from $[\mathrm{LaMc}]$ is more appropriate here.

\section{Relative $\mathbb{L}$-HOMOlOGy Classes}

Here we consider ordered simplicial complexes $B, K$ and a simplicial map $p$ : $K \rightarrow B$. We assume $B, K$ to be finite complexes, and as in $\S 3$ let $B \subset \partial \Delta^{m+1}$ be an order preserving embedding. By the simplicial mapping cylinder construction we substitute $p$ by an inclusion $K \subset B$. An element $[y] \in H_{n+1}(B, K, \mathbb{L})$ is given by a semi-simplicial map

$$
y:(\bar{K}, \bar{B}) \rightarrow\left(\mathbb{L}_{n+1-m}, \emptyset\right),
$$

i.e. by a family $\left\{y(\tau) \in \mathbb{L}_{n+1-m}(m-|\tau|)\right\}$.

Each $y(\tau)$ is a surgery problem $\left(f_{\tau}, b_{\tau}\right): W_{\tau} \rightarrow V_{\tau}$ of manifold $(m-|\tau|+3)$-ads with $\operatorname{dim} V_{\tau}=\operatorname{dim} W_{\tau}=n+1-|\tau|$, such that $f_{\tau}$ restricts to a homotopy equivalence

$$
\partial_{m-|\tau|+1} W_{\tau} \rightarrow \partial_{m-|\tau|+1} V_{\tau} .
$$

It is convenient to give the family $\{y(\tau)\}$ a "cobordism" interpretation: $y$ can be written as a semi-simplicial map:

$$
y:\left(\Delta^{1} \times \Sigma^{m}, \Delta^{1} \times \bar{B} \cup \partial_{0} \Delta^{1} \times \bar{K} \cup \partial_{1} \Delta^{1} \times \Sigma^{m}\right) \rightarrow\left(\mathbb{L}_{n-m}, \emptyset\right)
$$

(cf. [Ran, p. 128]).

The connecting homomorphism $\partial_{*}: H_{n+1}(B, K, \mathbb{L}) \rightarrow H_{n}(K, \mathbb{L})$ sends $[y]$ to $\partial_{*}[y]=[x]$, where

$$
x:\left(\partial_{0} \Delta^{1} \times \Sigma^{m}, \partial_{0} \Delta^{1} \times \bar{K}\right) \rightarrow\left(\mathbb{L}_{n-m}, \emptyset\right)
$$

is the restriction of $y$.

If we write $\Delta^{1} \times \sigma$ for $\Delta^{1} \times D\left(\sigma, \partial \Delta^{m+1}\right) \subset \Delta^{1} \times \Sigma^{m}$, considered as $(m-|\sigma|+$ 1) - ball, we have $y\left(\Delta^{1} \times \sigma\right) \in \mathbb{L}_{n-m}(m-|\sigma|+1)$; i.e. it is a surgery problem

$$
\left(f_{\Delta^{1} \times \sigma}, b_{\Delta^{1} \times \sigma}\right): W_{\Delta^{1} \times \sigma}^{n+1-|\sigma|} \rightarrow V_{\Delta^{1} \times \sigma}^{n+1-|\sigma|}
$$


between $(m-|\sigma|+5)$-ads, such that $W_{\Delta^{1} \times \sigma}, V_{\Delta^{1} \times \sigma}$ are $(n+1-|\sigma|)$-dimensional manifolds and $f_{\Delta^{1} \times \sigma}$ restricts to a homotopy equivalence

$$
\partial_{m-|\sigma|+3} W_{\Delta^{1} \times \sigma} \rightarrow \partial_{m-|\sigma|+3} V_{\Delta^{1} \times \sigma} .
$$

Note that $\partial_{0} V_{\Delta^{1} \times \sigma}=V_{\partial_{0} \Delta^{1} \times \sigma}, \partial_{0} W_{\Delta^{1} \times \sigma}=W_{\partial_{0} \Delta^{1} \times \sigma}$. Furthermore, we have

(I) $V_{\Delta^{1} \times \sigma} \neq \emptyset \Rightarrow \sigma \in B$;

(II) $\partial_{0} V_{\Delta^{1} \times \sigma} \neq \emptyset \Rightarrow \sigma \in K$; and

(III) $\partial_{1} V_{\Delta^{1} \times \sigma}=V_{\partial_{1} \Delta^{1} \times \sigma}=\emptyset$ for all $\sigma \in \partial \Delta^{m+1}$.

Hence $\left\{W_{\Delta^{1} \times \sigma} \rightarrow V_{\Delta^{1} \times \sigma}\right\}$ can be considered as the adic-surgery problem bounding the adic-surgery problem $\left\{\partial_{0} W_{\Delta^{1} \times \sigma} \rightarrow \partial_{0} V_{\Delta^{1} \times \sigma}\right\}$. The latter represents $\partial_{*}[y]=$ $[x] \in H_{n}(K, \mathbb{L})$.

Let $(f, b): W \rightarrow V$ be the colimit understood as in $\S 3$. From Proposition 6.6 in LaMc] we obtain a degree 1 normal map $(f, b)$ between $(n+1)$-manifolds with boundaries

$$
\partial V=\partial_{0} V \cup \partial^{\prime} V, \partial W=\partial_{0} W \cup \partial^{\prime} W,
$$

where $\partial_{0} V$ is the colimit of $x, \partial^{\prime} V$ is the colimit of the restrictions to all $\partial_{m-|\sigma|+3} V_{\Delta^{1} \times \sigma}$, and similarly for $\partial W$. Clearly, $f$ restricted to $\partial^{\prime} W$ is a homotopy equivalence. The colimit of the reference maps gives a reference map $\left(V, \partial_{0} V\right) \stackrel{\mathcal{S}}{\longrightarrow}(B, K)$.

We now describe the map $p_{*}: H_{n}(K, \mathbb{L}) \rightarrow H_{n}(B, \mathbb{L})$. Suppose that $[x]$ is represented by the normal degree 1 map $(f, b): M \rightarrow X$ with the reference map $\mathcal{S}: X \rightarrow K$. Then the family $M_{\sigma} \stackrel{f_{\sigma}, b_{\sigma}}{\longrightarrow} X_{\sigma}, \sigma \in K$, with $X_{\sigma}=\mathcal{S}^{-1}(D(\sigma, K))$, $M_{\sigma}=f^{-1}\left(X_{\sigma}\right), f_{\sigma}=\left.f\right|_{M_{\sigma}}$, defines a cycle $x$. Note that $p_{*}[x]$ is represented by the family $\widehat{M}_{\tau} \stackrel{\widehat{f}_{\tau}, \widehat{b}_{\tau}}{\longrightarrow} \widehat{X}_{\tau}, \tau \in B$, with $\widehat{X}_{\tau}=\widehat{\mathcal{S}}^{-1}(D(\tau, B)), \widehat{M}_{\tau}=f^{-1}\left(\widehat{X}_{\tau}\right), \widehat{f}_{\tau}=\left.f\right|_{\widehat{M}_{\tau}}$, where we have put $\widehat{\mathcal{S}}=\mathcal{S} \circ p$.

We also have to understand in this context the meaning of "homologies" between cycles $x, x^{\prime}$ on $K$, i.e. $[x]=\left[x^{\prime}\right] \in H_{n}(K, \mathbb{L})$. There is a semi-simplicial map

$$
y:\left(\Delta^{1} \times \Sigma^{m}, \Delta^{1} \times \bar{K}\right) \rightarrow\left(\mathbb{L}_{n-m}, \emptyset\right)
$$

such that $y\left(\partial_{0} \Delta^{1} \times \sigma\right)=x(\sigma), y\left(\partial_{1} \Delta^{1} \times \sigma\right)=x^{\prime}(\sigma)$, i.e.

$$
y\left(\Delta^{1} \times \sigma\right) \in \mathbb{L}_{n-m}(m-|\sigma|+1),
$$

defining surgery problems

$$
\left(F_{\Delta^{1} \times \sigma}, B_{\Delta^{1} \times \sigma}\right): W_{\Delta^{1} \times \sigma} \rightarrow V_{\Delta^{1} \times \sigma}
$$

of $(m-|\sigma|+5)$-ads as above, such that it restricts to

$$
\left(f_{\sigma}, b_{\sigma}\right): \partial_{0} W_{\Delta^{1} \times \sigma}=M_{\sigma} \rightarrow \partial_{0} V_{\Delta^{1} \times \sigma}=X_{\sigma}
$$

and

$$
f_{\sigma}^{\prime}, b_{\sigma}^{\prime}: \partial_{1} W_{\Delta^{1} \times \sigma}=M_{\sigma}^{\prime} \rightarrow \partial_{1} V_{\Delta^{1} \times \sigma}=X_{\sigma}^{\prime}
$$

being $x(\sigma)$ and $x^{\prime}(\sigma)$, respectively. Moreover, $F_{\Delta^{1} \times \sigma}$ restricts to a homotopy equivalence

$$
\partial_{m-|\sigma|+3} W_{\Delta^{1} \times \sigma} \rightarrow \partial_{m-|\sigma|+3} V_{\Delta^{1} \times \sigma} .
$$

The colimit construction gives a normal cobordism $(F, B): W^{n+1} \rightarrow V^{n+1}$ such that $\partial W=\partial_{0} W \cup \partial_{1} W \cup \partial^{\prime} W \rightarrow \partial_{0} V \cup \partial_{1} V \cup \partial^{\prime} V$ with $\partial^{\prime} W \rightarrow \partial^{\prime} V$ as a controlled homotopy equivalence extending the homotopy equivalences $\partial M \rightarrow \partial X$ and $\partial M^{\prime} \rightarrow$ $\partial X^{\prime}$. Moreover, $\partial_{0} W \rightarrow \partial_{0} V$ and $\partial_{1} W \rightarrow \partial_{1} V$ represent $x$ (resp. $x^{\prime}$ ) by normal degree 1 maps. 
We shall now consider the case of a closed topological $n$-manifold $K$. By the Poincaré duality (cf. Ran), we have

$$
H_{n}(K, \mathbb{L})=H^{0}(K, \mathbb{L})=[K, \mathbb{Z} \times G / T O P] .
$$

The $\mathbb{Z}$-sector is related to Quinn's index invariant. The simply connected cover $\mathbb{L}^{+} \rightarrow \mathbb{L}$ is characterized by $\mathbb{L}_{0}^{+}=G / T O P$, and induces an injection $[K, G / T O P] \rightarrow$ $[K, \mathbb{Z} \times G / T O P]$ onto the 1-sector. By duality it is the image of $H_{n}\left(K, \mathbb{L}^{+}\right) \rightarrow$ $H_{n}(K, \mathbb{L})$ (cf. Qu2, [Ran, §25], and [BFMW] for more details).

Given an element $[x] \in H_{n}\left(K, \mathbb{L}^{+}\right)$it defines a normal degree 1 map between closed manifolds

$$
\begin{gathered}
(g, c): Z^{n} \rightarrow K^{n} \text { giving a cycle representation } \\
\left\{z_{\sigma}\right\}=\left\{g_{\sigma}, c_{\sigma}\right\}: Z_{\sigma} \rightarrow K_{\sigma}=D(\sigma, K) \subset D\left(\sigma, \partial \Delta^{m+1}\right) .
\end{gathered}
$$

Obviously, $\partial_{m-|\sigma|+1} K_{\sigma}=\emptyset$, and the colimit of the family $\left\{K_{\sigma} \mid \sigma \in K\right\}$ gives $K$. The cycle $\left\{z_{\sigma}\right\}=z$ represents $[z]=[x] \in H_{n}\left(K, \mathbb{L}^{+}\right) \subset H_{n}(K, \mathbb{L})$. If $(f, b)$ : $M^{n} \rightarrow X^{n}$ is the normal degree 1 map obtained from the colimit of the cycle $x$, there is a normal cobordism $C^{n+1} \rightarrow Y^{n+1}$ with $\partial_{0} C^{n+1} \rightarrow \partial_{0} Y^{n+1}$ equal to $(f, b)$ and $\partial_{1} C^{n+1} \rightarrow \partial_{1} Y^{n+1}$ equal to $(g, c) . \partial_{0} Y \cup \partial_{1} Y \subset \partial Y, \partial_{0} C \cup \partial_{1} C \subset$ $\partial C$, and $\partial Y \backslash\left(\partial_{0} Y \cup \partial_{1} Y\right)$ have boundaries $\partial^{\prime} X=\partial X$ defined above. Similarly, $\partial C \backslash\left(\partial_{0} C \cup \partial_{1} C\right)$ have boundaries $\partial^{\prime} M=\partial M$.

We define $H_{n+1}^{+}(B, K, \mathbb{L}) \subset H_{n+1}(B, K, \mathbb{L})$ as the inverse image of $H_{n}\left(K, \mathbb{L}^{+}\right) \subset$ $H_{n}(K, \mathbb{L})$ under $\partial_{*}: H_{n+1}(B, K, \mathbb{L}) \rightarrow H_{n}(K, \mathbb{L})$. Obviously, $H_{n+1}\left(B, K, \mathbb{L}^{+}\right) \subset$ $H_{n+1}^{+}(B, K, \mathbb{L})$.

Finally, let a class $[y] \in H_{n+1}^{+}(B, K, \mathbb{L})$ be given with boundary cycle $x$, i.e. $[x]=\partial_{*}[y]$. Let

$$
W \rightarrow V, \partial_{0} W=M \stackrel{(f, b)}{\longrightarrow} \partial_{0} V=X
$$

be the colimits of $y$ and $x$. We glue $C \rightarrow Y$ to $W \rightarrow V$ along $M \stackrel{(f, b)}{\longrightarrow} X$ and obtain a normal degree 1 map:

$$
\begin{gathered}
\left(F^{+}, B^{+}\right): W^{+} \rightarrow V^{+} \text {with restriction } \\
(g, c): \partial_{0} W^{+}=Z \rightarrow \partial_{0} V^{+}=K, \partial^{\prime} W^{+}=\partial^{\prime} W, \partial^{\prime} V^{+}=\partial^{\prime} V, \\
\left(F^{+}, B^{+}\right) \text {defines an element }\left[y^{+}\right] \in H_{n+1}^{+}(B, K, \mathbb{L}) .
\end{gathered}
$$

By the normal cobordism extension property (cf. [Med, p. 45] or [Wal, p. 93] applied in the proof of Theorem 9.6), $W^{+} \rightarrow V^{+}$and $W \rightarrow V$ are normally cobordant by a cobordism being a product outside a small neighborhood of $C \rightarrow Y$.

In summary, we got for any closed oriented $n$-manifold $p: K \rightarrow B$ a continuous map into a simplicial complex and any $[y] \in H_{n+1}^{+}(B, K, \mathbb{L})$ a cycle representation $y$ with colimit a degree 1 normal map

$$
(f, b): W^{n+1} \rightarrow V^{n+1}
$$

between $(n+1)$-dimensional manifolds such that $K=\partial_{0} V \subset \partial V, f$ restricted to $\partial_{0} W=M \rightarrow K$ is the colimit of a cycle of $\partial_{*}[y] \in H_{n}(K, \mathbb{L})$. Moreover, $f$ restricted to $\partial W \backslash \partial_{0} W \rightarrow \partial V \backslash \partial_{0} V$ is a homotopy equivalence. It is a $\delta$-homotopy equivalence with respect to the reference map $\partial V \backslash \partial_{0} V \rightarrow B$.

A special case is given when $[y] \in H_{n+1}^{+}(B, K, \mathbb{L})$ is in the image of $i_{*}: H_{n+1}(B, \mathbb{L})$ $\rightarrow H_{n+1}^{+}(B, K, \mathbb{L})$, i.e. $i_{*}([z])=[y]$. In this case one gets a normal degree 1 map $W \rightarrow V$ with $\partial_{0} W \rightarrow \partial_{0} V=\emptyset$ and $\partial W=\partial^{\prime} W \rightarrow \partial^{\prime} V=\partial V$ a controlled homotopy equivalence. 
For our later use we substitute $W \rightarrow V$ by adding $C \rightarrow Y$ representing the trivial element in $H_{n+1}^{+}(B, K, \mathbb{L})$. We can take $C=K \times I=Y$, with $\partial_{0} C=K \times 0=\partial_{0} Y$, $\partial_{1} C=\partial_{1} Y=\emptyset$ and $\partial^{\prime} C=K \times 1=\partial^{\prime} Y$. We shall later refer to this case as the "absolute case" and write it as

$$
W \dot{\cup} C \rightarrow V \dot{\cup} Y .
$$

Moreover, we shall ignore the boundaries $\partial^{\prime} W, \partial^{\prime} V, \partial^{\prime} C$ and $\partial^{\prime} Y$.

$$
\text { 5. THE MAP } a: H_{n+1}^{+}(B, K, \mathbb{L}) \rightarrow \mathcal{S}_{\varepsilon, \delta}(K)
$$

We assume $K$ to be a closed oriented $n$-manifold, $p: K \rightarrow B$ a $U V^{1}$-map, and $B$ a compact finite-dimensional metric ANR. To define the map $a$ it suffices to take $B$ a finite ordered simplicial complex contained in $\partial \Delta^{m+1}$ as before.

We must first introduce the controlled structure set. Its definition comes with the proof of the controlled surgery sequence of $\mathrm{PQR}$ (cf. also [Fer]):

$$
H_{n+1}(B, \mathbb{L}) \rightarrow \mathcal{S}_{\varepsilon, \delta}(K \stackrel{p}{\longrightarrow} B) \rightarrow\left[K, G_{/ T O P}\right] \rightarrow H_{n}(B, \mathbb{L}) .
$$

The precise statement is: There exists $\varepsilon_{0}>0$ such that for any $0<\varepsilon<\varepsilon_{0}$ there is $\delta>0$ such that the sequence is exact provided $p$ is a $U V^{1}$-map $\left(U V^{1}(\delta)\right.$ would be sufficient). With these $\varepsilon$ and $\delta$, the controlled structure set $\mathcal{S}_{\varepsilon, \delta}(K \rightarrow B)$ can be defined: Elements in $\mathcal{S}_{\varepsilon, \delta}(K \rightarrow B)$ are represented by pairs $(M, f)$, where $M$ is a closed $n$-manifold and $f: M \rightarrow K$ is a $\delta$-homotopy equivalence with respect to the control map $p: K \rightarrow B$. Two pairs $\left(M_{1}, f_{1}\right)$ and $\left(M_{2}, f_{2}\right)$ are " $\varepsilon$ related" if there is a homeomorphism $h: M_{1} \rightarrow M_{2}$ such that $f_{2} \circ h$ and $f_{1}$ are $\varepsilon$-homotopic over $p: K \rightarrow B$ (i.e. the homotopy has "tracks" of size $<\varepsilon$ in $B$ ). Now, with this $\varepsilon$ and $f$, " $\varepsilon$-related" is an equivalence relation. We shall shortly write $\mathcal{S}_{\varepsilon, \delta}(K \stackrel{p}{\longrightarrow} B)=\mathcal{S}_{\varepsilon, \delta}(K)$.

Further remarks. In $[\mathrm{PQR}, \mathbb{L}$ denotes the algebraic $\mathbb{L}$-spectrum defined by adic quadratic Poincaré complexes (see [Ran]). To a normal degree 1-map $(f, b): M \rightarrow$ $K$ there is an obstruction in a controlled Wall group $L_{n}(B, \varepsilon, \delta)$. It consists of quadratic $n$-dimensional Poincaré complexes of radius $\delta>0$ over $B$, modulo $\varepsilon$ cobordism. If this obstruction vanishes, controlled surgery can be completed in the middle dimension. The controlled Hurewicz and Whitehead theorems give an element in $\mathcal{S}_{\varepsilon, \delta}(K)$. All this involves $\varepsilon$ - $\delta$-estimates. There is an assembly map $A$ : $H_{n}(B, \mathbb{L}) \rightarrow L_{n}(B, \varepsilon, \delta)$ defined by gluing the adic parts of an element in $H_{n}(B, \mathbb{L})$, similarly to how it was done in $\S 3$. This map is proved to be an isomorphism for suitable $\varepsilon, \delta$. It is here, among other places, where the "stability threshold" $\varepsilon_{0}>0$ drops in (see also RanYa]).

Let $[y] \in H_{n+1}^{+}(B, K, \mathbb{L})$ be given, and let $y$ be a cycle with $y(\sigma)$, given by normal degree $1(m-|\sigma|+5)$-ads $\left(f_{\sigma}, b_{\sigma}\right): W_{\sigma} \rightarrow V_{\sigma}$. Let $(f, b): W \rightarrow V$ be the colimit obtained in $\S 4$; i.e. $f$ restricts to a normal degree 1 map between closed manifolds $\partial_{0} W \rightarrow K$, and to a $\delta^{\prime}$-homotopy equivalence $\partial^{\prime} W \rightarrow \partial^{\prime} V$. We claim that $\delta^{\prime}$ can be chosen smaller than $\delta$. First note that the above $\varepsilon_{0}>0$ depends on $B$ (and on $n$ ), and $\delta^{\prime}$ becomes smaller and smaller if we subdivide $B$ finer and finer.

Let $\mathcal{S}: V \rightarrow B$ be the reference map. Restricting $\mathcal{S}$ to $\partial_{0} V=K$ gives $p: K \rightarrow B$, and by assumption it is $U V^{1}$.

We can assume that $S: V \rightarrow B$ is also $U V^{1}$ : First, change $W \rightarrow V$ as in the proof of Theorem 9.4 in Wal] to obtain an isomorphism $S_{*}: \pi_{1}(V) \rightarrow \pi_{1}(B)$. 
Then since $\operatorname{dim} V \geq 5$ one can approximate $S$ (rel. $\partial V$ ) to become a $U V^{1}$-map (cf. [BFMW, Theorem 4.4]).

We can invoke the controlled simply connected $\pi-\pi$-theorem (cf. e.g. [Fer]): We have a $\delta^{\prime}$-equivalence $\partial_{0} W \rightarrow \partial_{0} V=K$, and we can assume that $\partial^{\prime} W \rightarrow \partial^{\prime} V$ is a $\delta^{\prime}$-equivalence too, and $\delta^{\prime}$ can be as small as we need it to be. By the $\pi-\pi$-theorem there are $\delta_{0}>0, k>0$, such that for $\delta^{\prime}<\delta_{0}$ there is a normal cobordism between $\left(W, \partial_{0} W\right) \rightarrow\left(V, \partial_{0} V\right)$ relative $\partial^{\prime} V$ to a $k \delta^{\prime}$-homotopy equivalence

$$
f^{\prime}:\left(W^{\prime}, \partial_{0} W^{\prime}\right) \rightarrow\left(V, \partial_{0} V=K\right)
$$

Choosing further $k \delta^{\prime}<\delta$, we get a class in $\mathcal{S}_{\varepsilon, \delta}(K)$ represented by

$$
f_{0}^{\prime}: \partial_{0} W^{\prime} \rightarrow K, f_{0}^{\prime}=\left.f^{\prime}\right|_{\partial_{0} W^{\prime}} .
$$

We shall define $a: H_{n+1}^{+}(B, K, \mathbb{L}) \rightarrow \mathcal{S}_{\varepsilon, \delta}(K)$ by $a([y])=\left[f_{0}^{\prime}, \partial_{0} W^{\prime}\right]$.

It remains to show that $a$ is well-defined. Suppose $y, y_{1}$ are cycles with $\left[y_{1}\right]=[y]$; let

$$
\begin{gathered}
\left(W, \partial_{0} W\right) \stackrel{(f, b)}{\longrightarrow}\left(V, \partial_{0} V=K\right) \\
\left(W_{1}, \partial_{0} W_{1}\right) \stackrel{\left(f_{1}, b_{1}\right)}{\longrightarrow}\left(V_{1}, \partial_{0} V_{1}=K\right)
\end{gathered}
$$

be colimits and let

$$
\begin{gathered}
\left(W^{\prime}, \partial_{0} W^{\prime}\right) \stackrel{f^{\prime}}{\longrightarrow}\left(V, \partial_{0} V=K\right) \\
\left(W_{1}^{\prime}, \partial_{0} W_{1}^{\prime}\right) \stackrel{f_{1}^{\prime}}{\longrightarrow}\left(V_{1}, \partial_{0} V_{1}=K\right)
\end{gathered}
$$

be $k \delta^{\prime}$-homotopy (resp. $k \delta_{1}^{\prime}$-homotopy) equivalences obtained from the controlled $\pi-\pi$-theorem. We may assume $k \delta^{\prime}<\delta, k \delta_{1}^{\prime}<\delta$.

Let $Q \rightarrow \Omega$ be a normal cobordism between $(f, b)$ and $\left(f_{1}, b_{1}\right)$. It restricts to a normal cobordism $P \rightarrow K \times I$ with $\partial P=M \cup M_{1} \stackrel{f \cup f_{1}}{\longrightarrow} K \times\{0,1\}$, and with boundary $\partial Q=W \cup P \cup W_{1} \rightarrow \partial \Omega=V \cup K \times I \cup V_{1}$.

To proceed we briefly have to recall the proof of the $\pi-\pi$-theorem: The first step is to make $\left(W, M=\partial_{0} W\right) \rightarrow\left(V, \partial_{0} V=K\right)$ highly connected, which we can assume.

The second step consists of handle subtraction in the pairs $(W, M)$ and $\left(W_{1}, M_{1}\right)$, i.e. $\left(W^{\prime}, \partial_{0} W^{\prime}\right)$ is obtained from $W$ by subtracting handles. We add these handles to $P$ along $M$. Similarly, we add the handles subtracted from $\left(W_{1}, M_{1}\right)$ along $M_{1}$ to $P_{1}$. One obtains $P^{\prime}$ with $\partial P^{\prime}=M^{\prime} \cup M_{1}^{\prime}$. In other words, we obtain a new decomposition of $\partial Q$ and $\partial \Omega$ and $\partial Q \rightarrow \partial \Omega$ as

$$
\partial Q=W^{\prime} \cup P^{\prime} \cup W_{1}^{\prime} \rightarrow \partial \Omega=V \cup K \times I \cup V_{1} .
$$

Hence $Q \rightarrow \Omega$ displays a normal bordism between

$$
\left(P^{\prime}, \partial P^{\prime}=M^{\prime} \cup M_{1}^{\prime}\right) \rightarrow(K \times I, K \times\{0\} \cup K \times\{1\})
$$

and the controlled homotopy equivalence

$$
\left(W^{\prime}, M^{\prime}\right) \cup\left(W_{1}^{\prime}, M_{1}^{\prime}\right) \rightarrow(V, K) \cup\left(V_{1}, K\right) .
$$

We denote by $\phi^{\prime}: P^{\prime} \rightarrow K \times I$ the first map. Then $\phi^{\prime}$ restricted to $M^{\prime}, M_{1}^{\prime}$ is a $k \delta^{\prime}$ (resp. $k \delta_{1}^{\prime}$ ) equivalence, with $k \delta^{\prime}, k \delta_{1}^{\prime}<\delta$.

Let us assume $\delta_{1}^{\prime}=\delta^{\prime}$. Controlled surgery theory then implies (cf. PQR, Qu1, [Fer]) that $\phi^{\prime}$ can be surgered to a simple $\bar{k} k_{1} \delta^{\prime}$-homotopy equivalence

$$
H:\left(P^{\prime \prime}, M^{\prime}, M_{1}^{\prime}\right) \rightarrow(K \times I, K \times\{0\}, K \times\{1\})
$$

(for some $\bar{k}>0$ ) with $\left.H\right|_{M^{\prime}}=f^{\prime},\left.H\right|_{M_{1}^{\prime}}=f_{1}^{\prime}$. In particular $P^{\prime \prime}$ is an $s$-cobordism. 
Let $G: P^{\prime \prime} \rightarrow M^{\prime} \times[0,1]$ be a homeomorphism with

$$
\left.G\right|_{M^{\prime}}=I d, g=\left.G\right|_{M_{1}^{\prime}}: M_{1}^{\prime} \rightarrow M \text {. }
$$

The composition

$$
M_{1}^{\prime} \times I \stackrel{g \times I d}{\longrightarrow} M^{\prime} \times I \stackrel{G^{-1}}{\longrightarrow} P^{\prime \prime} \stackrel{H}{\longrightarrow} K \times I
$$

restricts to $f^{\prime} \circ g: M_{1}^{\prime} \times 0 \rightarrow K \times 0$ and $f_{1}^{\prime}: M_{1}^{\prime} \times\{1\} \rightarrow K \times\{1\}$. Therefore $f^{\prime} \circ g$ and $f_{1}^{\prime}$ are $\bar{k} k \delta^{\prime}$-homotopic over $B$.

However, we can choose $\delta^{\prime}$ such that $\bar{k} k \delta^{\prime}<\varepsilon$, showing $f^{\prime}: M^{\prime} \rightarrow K, f_{1}^{\prime}: M_{1}^{\prime} \rightarrow$ $K$ define the same element in $\mathcal{S}_{\varepsilon, \delta}(K)$; hence the map $a$ is well-defined.

This finishes the generic case, and we shall now come to the absolute case. Now let $[y] \in H_{n+1}^{+}(B, K, \mathbb{L})$ be the image of $[z] \in H_{n+1}^{+}(B, \mathbb{L})$, i.e. $i_{*}[z]=[y]$. Then we get a normal degree 1 map $W \dot{\cup} C \rightarrow V \dot{\cup} Y$ such that $\partial_{0} V=\emptyset, \partial_{0} W=\emptyset, C \rightarrow Y$ is a controlled homotopy equivalence with $\partial C=K \rightarrow \partial Y=K$ a homeomorphism. The $\pi-\pi$-theorem cannot be applied to $\left(W, \partial_{0} W\right)$ since $\partial_{0} W=\emptyset$. We define $a([y])$ as the result of the controlled Wall action of $[z]$ on $I d: K \rightarrow K$; i.e. $a[y]$ is the image of $[z]$ under

$$
H_{n+1}(B, \mathbb{L}) \rightarrow \mathcal{S}_{\varepsilon, \delta}(K)
$$

(cf. $\mathrm{PQR}$ ). We have to show that the map $a$ is well-defined. It suffices to consider the following two types of cycle representations of $i_{*}([z])=[y]$ :

(I) $W \dot{\cup} C \rightarrow V \dot{\cup} Y$ is an absolute representation;

(II) $W_{1} \rightarrow V_{1}$ is a generic representation; with $\partial_{0} W_{1} \rightarrow \partial_{0} V_{1} \neq \emptyset$.

Let $Q \rightarrow \Omega$ be a normal cobordism between them. Then $\partial Q=W \cup \dot{\cup} \cup \cup_{K} P \cup W_{1} \rightarrow$ $\partial \Omega=V \dot{\cup} Y \cup_{K} K \times I \cup V_{1}$, where $\left(P, \partial_{0} P=K, \partial_{1} P=\partial_{0} W_{1}\right) \rightarrow(K \times I, K \times 0, K \times 1)$.

The restriction to $W_{2}=C \cup P \cup W_{1} \rightarrow V_{2}=Y \cup K \times I \cup V_{1}$ is a normal degree 1 map with controlled homotopy equivalence on the boundary. It defines the same element $i_{*}[z]=[y] \in H_{n+1}^{+}(B, K, \mathbb{L})$. We may also cancel $C \rightarrow Y$ thus obtaining

$$
\begin{gathered}
W_{3}=P \cup W_{1} \rightarrow V_{3} \cong V_{1}, \text { and identity on } \\
\partial_{0} W_{3}=K \rightarrow \partial_{0} V_{3}=K .
\end{gathered}
$$

It still defines the same element $[y]$. We can apply the $\pi-\pi$-theorem to $\left(W_{3}, \partial_{0} W_{3}=K\right) \rightarrow\left(V_{3}, \partial_{0} V_{3}=K\right)$ and obtain a controlled homotopy equivalence

$$
\left(W_{3}^{\prime}, \partial_{0} W_{3}^{\prime}\right) \rightarrow\left(V_{3}, \partial_{0} V_{3}=K\right) .
$$

We have to show that the $\delta$-homotopy equivalence $\partial_{0} W_{3}^{\prime} \rightarrow K$ defines the same element as the image of

$$
[z] \in H_{n+1}(B, \mathbb{L}) \rightarrow \mathcal{S}_{\varepsilon, \delta}(K) .
$$

First, one makes $W_{3} \rightarrow V_{3}$ highly connected by surgeries in the interior of $W_{3}$. We shall assume this for $W_{3} \rightarrow V_{3}$. Assume $n+1=2 k$. The middle-dimensional classes defining the surgery kernel can be represented by framed immersed $(k+1)$ handles

$$
\left(H^{\prime}, \partial H^{\prime}\right) \rightarrow\left(W_{3}, K\right) \text {. }
$$

Let $U=K \times[0, \rho] \cup H^{\prime} \subset W_{3}$, where $K \times[0, \rho]$ denotes a small collar of $K$. Note that $U$ can be identified with the controlled Wall action of $[z]$ on $K$. On the other hand one can remove the intersection of the immersed handles across $K$ (here $\pi_{1}(K) \cong \pi_{1} W_{3}$ is used) to get embedded handles $(H, \partial H) \subset\left(W_{3}, K\right)$. 
We may assume that $U=K \times[o, \rho] \cup H$. Then $\left(W_{3}^{\prime}, \partial_{0} W_{3}^{\prime}\right)=\left(W_{3} \backslash \stackrel{\circ}{U}, \partial_{1} U\right) \rightarrow$ $\left(V_{3}, K\right)$ is the result from the $\pi-\pi$-theorem. This shows that both definitions of the map $a$ on $i_{*}[z]=[y]$ give the same result.

The case when $n+1$ is odd goes as follows: One has to consider neighbourhoods $U$ of $K$ in $W_{3}$ given by attached embedded handles of the type $S^{k} \times D^{k+1}, 2 k+1=$ $n+1$. They have to be subtracted. On the other hand, in order to realize an element of $H_{n+1}(B, \mathbb{L})$ one attaches trivial handles and then applies the controlled obstruction to the transverse spheres $1 \times S^{k}$. On this result one then performs the surgeries. The resulting homotopy equivalence is the same.

\section{Proof of Theorem 3}

The map $b$ was defined in DrFe as the composite map

$$
H_{n+1}(B, K, \mathbb{L}) \cong S_{n+1}(B, K, \mathbb{L}) \stackrel{\partial_{*}}{\longrightarrow} S_{n}(K, \mathbb{L}) \rightarrow \mathcal{S}(K) .
$$

Here, $S_{n+1}(B, K, \mathbb{L}), S_{n}(K, \mathbb{L})$ are the structure sets defined in Ran (however, denoted differently as $\left.S_{n+2}(B, K), S_{n+1}(K)\right)$.

An element in $S_{n}(K, \mathbb{L})$ is represented by a homotopy equivalence of $n$-manifolds $P^{n} \rightarrow Q^{n}$ with the reference map $Q^{n} \rightarrow K$. There is a natural inclusion $\mathcal{S}(K) \subset$ $S_{n}(K, \mathbb{L})$. The first isomorphism is due to the $\pi-\pi$-theorem. The map $S_{n}(K, \mathbb{L}) \rightarrow$ $\mathcal{S}(K)$ is such that the composite $\mathcal{S}(K) \subset S_{n}(K, \mathbb{L}) \rightarrow \mathcal{S}(K)$ is the identity.

Let $[y] \in H_{n+1}^{+}(B, K, \mathbb{L})$. Our construction of $a[y]$ produces a homotopy equivalence $\left(W^{\prime}, \partial_{0} W^{\prime}\right) \rightarrow\left(V, \partial_{0} V=K\right)$ with the reference map $\left(V, \partial_{0} V=K\right) \rightarrow(B, K)$.

This is the image of $[y]$ in $S_{n+1}(B, K, \mathbb{L})$; hence $\partial_{0} W^{\prime} \rightarrow \partial_{0} V$ is the image in $S_{n}(K, \mathbb{L})$. However, it belongs already to $\mathcal{S}(K) \subset S_{n}(K, \mathbb{L})$, and $\mathcal{S}(K) \subset$ $S_{n}(K, \mathbb{L}) \rightarrow \mathcal{S}(K)$ is the identity. This shows that the diagram

$$
\begin{array}{cccc}
H_{n+1}(B, K, \mathbb{L}) \cong S_{n+1}(B, K, \mathbb{L}) & \rightarrow \quad S_{n}(K, \mathbb{L}) & \rightarrow & \mathcal{S}(K) \\
\cup & & & \uparrow \\
H_{n+1}^{+}(B, K, \mathbb{L}) & & & \rightarrow \mathcal{S}_{\varepsilon, \delta}(K \rightarrow B)
\end{array}
$$

commutes.

To complete the proof of Theorem 3 we have to verify the commutativity of the diagrams

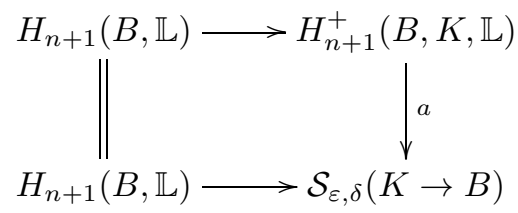

and

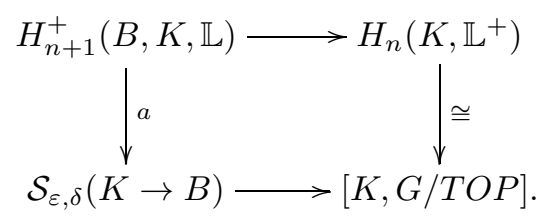


Now, (I) follows from the definition. To prove commutativity of (II), we consider the big diagram

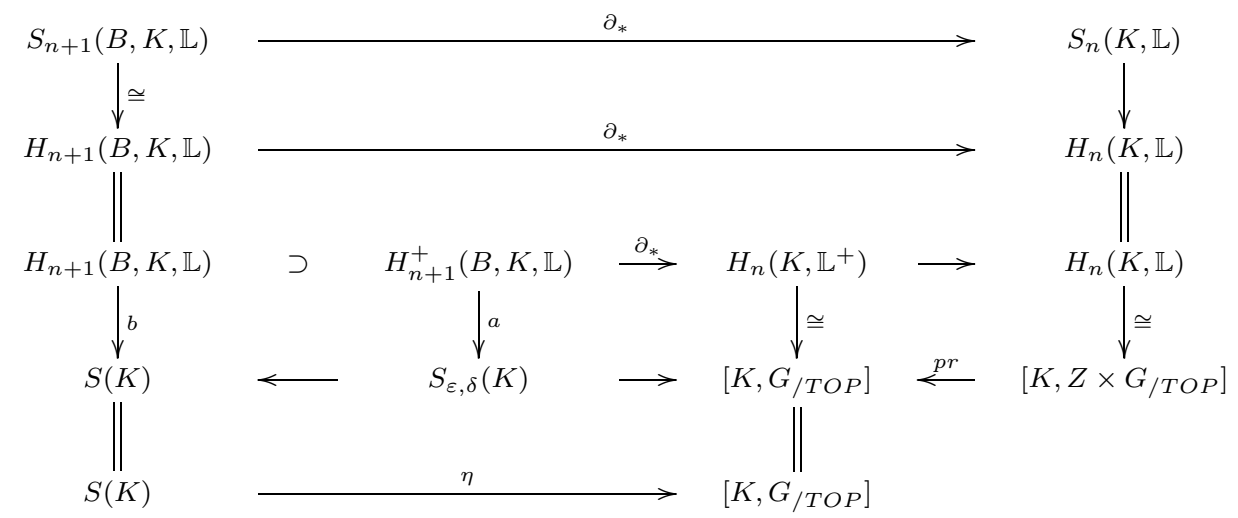

The "inner" diagram is (II). The upper 3 rows are just definitions of various $\partial_{*}$ (see also Ran, p. 207). Commutativity of the middle left diagram is explained in the above diagram. The middle right diagram commutes because of compatibility of $\mathbb{L}$ and $\mathbb{L}^{+}$-Poincaré duality. The lower diagram commutes; here $\eta$ associates to a homotopy equivalence $f: M \rightarrow K$ its normal invariant, and $p r$ denotes the projection map. The composed maps of the "outer" diagram give the diagram

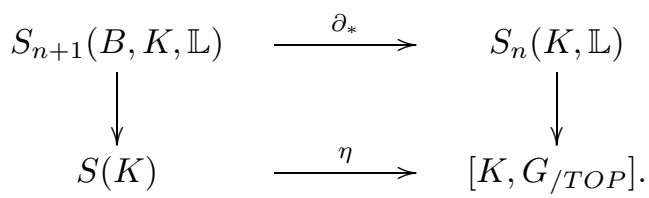

This commutes because of commutativity of

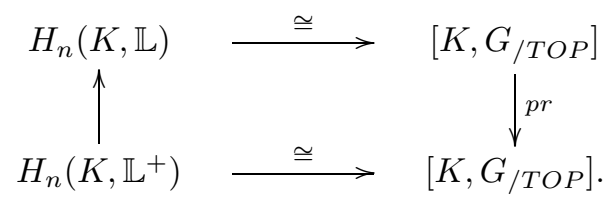

Commutativity of (II) follows from commutativity of all these diagrams.

\section{ACKNOWLEDGEMENTS}

This research was supported by the Slovenian Research Agency grants P1-02920101 and J1-4144-0101. The authors thank the referee for comments.

\section{REFERENCES}

[BFMW] J. Bryant, S. Ferry, W. Mio, and S. Weinberger, Topology of homology manifolds, Ann. of Math. (2) 143 (1996), no. 3, 435-467, DOI 10.2307/2118532. MR1394965(97b:57017)

[BRS] S. Buoncristiano, C. P. Rourke, and B. J. Sanderson, A geometric approach to homology theory, London Mathematical Society Lecture Note Series, No. 18, Cambridge University Press, Cambridge, 1976. MR0413113(54 \#1234)

[Coh] Marshall M. Cohen, Simplicial structures and transverse cellularity, Ann. of Math. (2) 85 (1967), 218-245. MR0210143 (35 \#1037)

[DrFe] A. N. Dranishnikov and S. Ferry, Cell-like maps and topological structure groups and manifolds, arXiv:math/0611004v1 [math GT] 31 Oct 2006. 
[Fer] Steven C. Ferry, Epsilon-delta surgery over $\mathbb{Z}$, Geom. Dedicata 148 (2010), 71-101, DOI 10.1007/s10711-009-9405-y. MR2721620 (2011m:57030)

[Ham] I. Hambleton, Surgery obstructions on closed manifolds and the inertia subgroup, Forum Math. 24 (2012), no. 5, 911-929. MR2988567

[Hat] Allen Hatcher, Algebraic topology, Cambridge University Press, Cambridge, 2002. MR.1867354 (2002k:55001)

[LaMc] G. Laures and J. McClure, Multiplicative properties of Quinn spectra, Forum Mathematicum, DOI 10.1515/forum-2011-0086

[Med] S. López de Medrano, Involutions on manifolds, Ergebnisse der Mathematik und ihrer Grenzgebiete, Band 59, Springer-Verlag, New York, 1971. MR0298698 (45 \#7747)

[Nic] Andrew J. Nicas, Induction theorems for groups of homotopy manifold structures, Mem. Amer. Math. Soc. 39 (1982), no. 267, vi+108. MR668807 (83i:57026)

[PQR] Erik Kjær Pedersen, Frank Quinn, and Andrew Ranicki, Controlled surgery with trivial local fundamental groups, High-dimensional manifold topology, World Sci. Publ., River Edge, NJ, 2003, pp. 421-426, DOI 10.1142/9789812704443_0018. MR.2048731 (2005e:57077)

[Qu1] Frank Quinn, A geometric formulation of surgery, Topology of Manifolds (Proc. Inst., Univ. of Georgia, Athens, Ga., 1969), Markham, Chicago, Ill., 1970, pp. 500-511. MR0282375 (43 \#8087)

[Qu2] Frank Quinn, An obstruction to the resolution of homology manifolds, Michigan Math. J. 34 (1987), no. 2, 285-291, DOI 10.1307/mmj/1029003559. MR894878 (88j:57016)

[Ran] A. A. Ranicki, Algebraic L-theory and topological manifolds, Cambridge Tracts in Mathematics, vol. 102, Cambridge University Press, Cambridge, 1992. MR.1211640 (94i:57051)

[RanYa] Andrew Ranicki and Masayuki Yamasaki, Controlled L-theory, Exotic homology manifolds-Oberwolfach 2003, Geom. Topol. Monogr., vol. 9, Geom. Topol. Publ., Coventry, 2006, pp. 105-153, DOI 10.2140/gtm.2006.9.105. MR2222493 (2007d:57048)

[Wal] C. T. C. Wall, Surgery on compact manifolds, London Mathematical Society Monographs, No. 1, Academic Press, London, 1970. MR0431216 (55 \#4217)

Department of Mathematics, University of Milano, Via C. Saldini 50, 02130 Milano, ITALY

E-mail address: friedrich.hegenbarth@mat.unimi.it

Faculty of Education, and Faculty of Mathematics and Physics, University of LjublJana, Kardeljeva Pl. 16, 1000 Ljubluana, Slovenia

E-mail address: dusan.repovs@guest.arnes.si 